\title{
Requisitos uniformes para preparar los manuscritos enviados a revistas biomédicas ${ }^{1}$
}

\author{
Comité Internacional de Directores de Revistas Médicas²
}

La quinta edición (1997) de los requisitos uniformes se ha preparado con la finalidad de reorganizar y modificar la redacción de la cuarta edi-
En 1978, un reducido grupo de directores de revistas médicas generales que se publican en inglés se reunió de manera informal en Vancouver (Canadá) a fin de fijar normas con respecto al formato que deberían adoptar los manuscritos enviados a esas publicaciones. Este fue el inicio de lo que con el tiempo llegó a conocerse como el Grupo de Vancouver. Sus requisitos para la preparación de manuscritos, que incluían el formato de las referencias bibliográficas creado por la Biblioteca Nacional de Medicina de los Estados Unidos, se publicaron por vez primera en 1979. Con el paso del tiempo el Grupo de Vancouver creció y se convirtió en el Comité Internacional de Directores de Revistas Médicas (CIDRM), que se reúne una vez al año y que gradualmente ha venido ampliando los temas que le conciernen.

El Comité ha producido cinco ediciones de los requisitos uniformes para preparar los manuscritos enviados a revistas biomédicas. A lo largo de los años han surgido asuntos que van más allá de la preparación del manuscrito. Algunos de esos asuntos se han incorporado a los requisitos uniformes, mientras que otros se tratan en declaraciones por separado. Cada declaración se ha publicado en una revista científica.

\footnotetext{
Traducción al español basada en: International Committee of Medical Journal Editors. Uniform requirements for manuscripts submitted to biomedical journals. Ann Intern Med 1997;126:36-47. La presente traducción y la de las declaraciones que el Comité Internacional de Directores de Revistas Médicas publica por separado (que se incluirán en otro número de esta revista) se podrán encontrar en el siguiente sitio de la World Wide Web: http://www.paho.org/spanish/DBI/ authors.htm

Traductor: Dr. Gustavo A. Silva, OPS, Washington, DC, EUA. Dirección postal: Organización Panamericana de la Salud, AGS/T, 525 Twentythird Street, Washington, DC 20037, EUA. Correo electrónico: 1silvagu@paho.org

El documento original en inglés se puede consultar en versión digital en diversos sitios de la World Wide Web, entre otros ACP Online (http:/www. acponline.org).
}

ción a efecto de aumentar la claridad y abordar inquietudes con respecto a derechos, protección de la vida privada de los sujetos de investigación,

Este documento no está protegido por derechos de autor. Puede copiarse o reimprimirse sin autorización, siempre y cuando se haga sin fines de lucro.

Las consultas y observaciones deben dirigirse a Kathleen Case, ICMJE Secretariat Office, Annals of Internal Medicine, American College of Physicians, Independence Mall West, Sixth Street at Race, Philadelphia, PA 19106-1572, EUA. Teléfono: 215351-2661; fax: 215-351-2644; correo electrónico: Kathyc@acp.mhs.compuserve.com

2 Actualmente integran el comité editores afiliados con las siguientes revistas y entidades: Annals of Internal Medicine, Biblioteca Nacional de Medicina de los Estados Unidos, British Medical Journal, Canadian Medical Association Journal, The Journal of the American Medical Association, The Lancet, The Medical Journal of Australia, The New England Journal of Medicine, The New Zealand Medical Journal, Tidsskrift for den Norske Laegeforening, Universidad de Princeton y Western Journal of Medicine. descripción de los métodos aplicados y otros asuntos. Los requisitos uniformes para preparar los manuscritos enviados a revistas biomédicas pueden reproducirse para fines educativos y sin afán de lucro, con prescindencia de los derechos de autor; el Comité alienta la distribución de este material.

A las revistas que accedan a guiarse por los requisitos uniformes (actualmente más de 500 revistas lo hacen) se les pide que en sus instrucciones para los autores citen el documento correspondiente a 1997.

Es importante hacer hincapié en lo que los requisitos significan y en lo que no. En primer lugar, los requisitos uniformes son instrucciones sobre la forma de preparar los manuscritos 
dirigidas a los autores; no se trata de indicaciones sobre estilo editorial destinadas a la redacción de las revistas. (Sin embargo, muchas revistas han incorporado en su estilo editorial ciertos elementos de los requisitos.)

En segundo lugar, si los autores preparan sus manuscritos según el estilo especificado en estos requisitos, los directores de las revistas participantes no devolverán los manuscritos para que se hagan cambios de estilo antes de considerarlos para publicación. No obstante, en el proceso de publicación los manuscritos podrán ser modificados por las redacciones de las revistas para adaptarlos a las particularidades de su propio estilo editorial.

En tercer lugar, los autores que envíen manuscritos a una revista participante se abstendrán de prepararlos de acuerdo con el estilo editorial de esta y se concretarán a cumplir con los requisitos uniformes.

Pese a todo, los autores tendrán asimismo que seguir las instrucciones particulares de la revista en cuestión por lo que se refiere a los temas apropiados para esta y el tipo de manuscritos que se le pueden enviar: por ejemplo, artículos originales, revisiones o informes de casos. Además, es probable que en dichas instrucciones figuren otros requisitos exclusivos de la publicación, tales como el número de copias del manuscrito, los idiomas en que este puede ser redactado, la extensión de los artículos y las abreviaturas aprobadas.

Se espera que las revistas participantes declaren en sus instrucciones a los autores que sus normas están de acuerdo con los requisitos uniformes para preparar los manuscritos enviados a revistas biomédicas y citen una versión publicada de estos.

\section{CUESTIONES QUE DEBEN CONSIDERARSE ANTES DE PRESENTAR UN MANUSCRITO PARA PUBLICACIÓN}

\section{Publicación redundante o duplicada}

Por publicación redundante o duplicada se entiende la publicación de un artículo que se traslapa considerablemente con otro ya publicado.

Los lectores de publicaciones periódicas primarias merecen que se les dé la confianza de que lo que están leyendo es original, a menos que haya una clara indicación de que el artículo se ha vuelto a publicar por elección del autor y el director. Esta posición tiene como fundamento las leyes internacionales de derechos de autor, la conducta ética y el uso eficaz en función de los costos de los recursos.

La mayor parte de las revistas científicas no desean recibir manuscritos acerca de un trabajo que ya se ha dado a conocer en gran medida en un artículo publicado o que forma parte de otro manuscrito que se ha propuesto o ha sido aceptado para publicación en otra parte, ya sea en forma impresa o en soporte electrónico. Esta norma no impide que la revista considere un artículo rechazado por otra revista o un informe completo que sigue a la publicación de un informe preliminar, como puede ser un resumen o póster presentados a la consideración de colegas en una reunión profesional. Tampoco impide que las revistas consideren un artículo que se ha presentado en una reunión científica pero que no se ha publicado íntegramente ni se está considerando para publicación en las actas de una reunión o en una publicación semejante. Las informaciones periodísticas acerca de reuniones programadas no se considerarán en general como infracciones de esta regla, pero no habrán de ampliarse mediante datos suplementarios o copias de los cuadros y las ilustraciones.

Cuando se envíe un artículo para publicación, el autor debe siempre adjuntar una relación completa de toda presentación del documento a otras revistas y de cualquier informe anterior que pudieran considerarse publicación redundante o duplicada del mismo trabajo o de uno muy semejante. El autor debe poner sobre aviso al director de la revista si el trabajo aborda temas sobre los cuales se hayan publicado informes anteriores. Si tal es el caso, esos trabajos se mencionarán con la debida referencia bibliográfica en el artículo nuevo. Junto con el manuscrito propuesto se incluirán copias de dichos materiales para ayudar al director a decidir la manera de manejar este asunto.

Si la publicación redundante o duplicada se intenta o se produce sin que medie la notificación descrita, los autores deberán atenerse a las medidas editoriales que se tomen en su contra. Como mínimo, cabe esperar el pronto rechazo del manuscrito presentado. Si el director no estaba al tanto de las infracciones y el artículo acaba por aparecer en su revista, entonces probablemente se publique en esta un aviso de publicación redundante o duplicada, que puede o no acompañarse de una explicación del autor y no necesitará de su aprobación.

La divulgación preliminar, generalmente por conducto de los medios de comunicación de masas, de la información científica descrita en un artículo ya aceptado pero aún sin publicar representa una infracción de las normas de muchas revistas. En contadas ocasiones, y solo mediante acuerdo con el director, puede aceptarse la diseminación preliminar de datos; por ejemplo, cuando se presenta una emergencia de salud pública.

\section{Publicación secundaria aceptable}

La publicación secundaria en el mismo idioma o en otro distinto, especialmente en otros países, se considera justificable y puede incluso ser beneficiosa, siempre y cuando se cumplan las siguientes condiciones.

1. Los autores tendrán que recabar la aprobación de los directores de las dos revistas involucradas; el director de la publicación secundaria tendrá en su poder una fotocopia, separata o manuscrito de la versión primaria.

2. Se respetará la precedencia de la publicación primaria dejando transcurrir por lo menos una semana antes de la publicación secundaria (a menos que específicamente se negocie otra cosa con ambos directores).

3. El artículo para publicación secundaria se destinará a un 
grupo diferente de lectores; podría bastar con una versión abreviada.

4. La versión secundaria reflejará fielmente los datos y las interpretaciones de la primaria.

5. Mediante una nota colocada al pie de la primera página de la versión secundaria, se informará a los lectores, a los colegas de los autores y a los organismos de documentación que el artículo ya se ha publicado con anterioridad en forma total o parcial, indicando la referencia primaria. Este podría ser un texto apropiado para dicha nota: "El presente artículo está basado en un estudio que se dio a conocer primero en [título de la revista y referencia completa]".

El permiso para la publicación secundaria de este tipo se otorgará gratuitamente.

\section{Protección de los derechos del paciente a que se respete su vida privada}

El derecho de los pacientes a que se respete su vida privada no debe ser infringido sin antes obtener su consentimiento fundamentado. Las descripciones por escrito, las fotografías y los árboles genealógicos que se publiquen no deberán contener información por la cual se pueda identificar a los pacientes, a menos que dichos datos resulten esenciales para fines científicos y que el paciente (o su padre o tutor) otorgue por escrito su autorización para la publicación después de haber sido debidamente informado. Para obtener el consentimiento para esta finalidad, es preciso mostrarle al paciente el manuscrito que se va a publicar.

Los detalles que puedan revelar la identidad del paciente deben omitirse si no son esenciales, pero los datos del paciente nunca se alterarán ni falsificarán solamente por tratar de lograr el anonimato. Es difícil lograr el anonimato completo, y frente a cualquier duda será preciso obtener el consentimiento fundamentado. Por ejemplo, cubrir la región de los ojos en las fotografías de los pacientes es una protección insuficiente del anonimato.

La exigencia del consentimiento con conocimiento de causa debe figurar en las instrucciones para los autores de la revista. Siempre que se obtenga dicha anuencia, esto deberá constar en el artículo publicado.

\section{REQUISITOS PARA LA PRESENTACIÓN DE MANUSCRITOS A UNA REVISTA}

\section{Resumen de los requisitos técnicos}

- Todas las partes del manuscrito estarán a doble espacio.

- Cada sección o componente comenzará en página nueva.

- Revise la secuencia: página del título, resumen y palabras clave, texto, agradecimientos, referencias, cuadros (cada uno en página aparte), pies o epígrafes de las ilustraciones.

- Las ilustraciones se presentarán en forma de impresiones fotográficas sin montar, y no deberán exceder de $203 \times 254 \mathrm{~mm}$.

- Incluya la autorización para reproducir material publicado con anterioridad o para usar ilustraciones en las que se pueda identificar a los sujetos humanos.

- Adjunte la transferencia de los derechos de autor y otros formularios.

- Presente el número exigido de copias impresas del artículo.

- Guarde copias de todo lo que envíe.

\section{Preparación del manuscrito}

El texto de los artículos de observación y experimentales se divide generalmente, aunque no por fuerza, en secciones que llevan estos encabezamientos: introducción, métodos, resultados y discusión. En los artículos largos puede ser necesario agregar subtítulos dentro de estas secciones, sobre todo en las de resultados y discusión, a fin de hacer más claro el contenido. Es probable que otro tipo de artículos —como los informes de casos, las revisiones y los editorialesexijan otra estructura. Para mayor orientación, los autores deberán consultar la revista en la que pretenden publicar.

Mecanografíese el manuscrito en papel bond blanco de $216 \times 279 \mathrm{~mm}$ o de la medida estándar ISO A4 (212 × $297 \mathrm{~mm})$, con márgenes de por lo menos $25 \mathrm{~mm}$. Escríbase o imprímase solamente sobre una cara del papel. Utilícese doble espacio a lo largo de todo el manuscrito, incluidos la página del título, el resumen, el texto, los agradecimientos, las referencias, cada uno de los cuadros y los pies o epígrafes de las ilustraciones. Numérense las páginas en forma consecutiva, empezando por la del título. Sobre el ángulo superior o inferior derecho de cada página anótese el número que le corresponde.

\section{Manuscritos en disquete}

Cuando un artículo se halla cercano a la aceptación definitiva, algunas revistas piden que los autores faciliten una copia en forma electrónica (en disquete); pueden aceptar una variedad de formatos de procesadoras de texto o ficheros de texto (ASCII).

Cuando presenten disquetes, los autores deberán:

1) cerciorarse de incluir la versión impresa del artículo que va en el disquete;

2) poner en el disquete únicamente la versión más reciente del manuscrito;

3) denominar claramente el fichero;

4) rotular el disquete con indicación del formato y el nombre del fichero;

5) facilitar información sobre el equipo de computación y el software utilizados.

Los autores deberán consultar las instrucciones que la revista proporciona a los autores para determinar cuáles son los formatos que se aceptan, las convenciones que se aplican para denominar los ficheros, el número de copias que deben presentarse y otros detalles. 


\section{Página del título}

La primera página contendrá: 1) el título del artículo, que será conciso pero informativo; 2) nombre de pila preferido y apellidos de cada autor, acompañados de sus grados académicos más importantes y su afiliación institucional; 3) nombre del departamento o departamentos y la institución o instituciones a los que se debe atribuir el trabajo; 4) declaraciones de descargo de responsabilidad, si las hay; 5) nombre y dirección del autor que se ocupará de la correspondencia relativa al manuscrito; 6) nombre y dirección del autor a quien se dirigirán las solicitudes de separatas, o nota informativa de que los autores no las proporcionarán; 7) procedencia del apoyo recibido en forma de subvenciones, equipo, medicamentos o todo ello; y 8) título abreviado (titulillo) que no pase de 40 pulsaciones (contando caracteres y espacios), el cual se colocará, debidamente identificado como tal, en la última línea de la página inicial.

\section{Autoría}

Todas las personas designadas como autores habrán de cumplir con ciertos requisitos para tener derecho a la autoría. Cada autor debe haber participado en el trabajo en grado suficiente para asumir responsabilidad pública por su contenido.

Para concederle a alguien el crédito de autor, hay que basarse únicamente en su contribución esencial por lo que se refiere a los siguientes aspectos: 1) la concepción y el diseño o bien el análisis y la interpretación de los datos; 2) la redacción del artículo o la revisión crítica de una parte importante de su contenido intelectual; y 3) la aprobación final de la versión que será publicada. Las tres condiciones tendrán que cumplirse siempre. La participación que consiste meramente en conseguir financiamiento o recoger datos no justifica el crédito de autor. Tampoco basta con ejercer la supervisión general del grupo de investigación. Toda parte del artículo que sea decisiva con res- pecto a las conclusiones principales deberá ser responsabilidad de por lo menos uno de los autores.

Los directores de revista podrán solicitar a los autores que describan la contribución de cada uno; esa información puede ser publicada.

Cada vez es más común que los ensayos multicéntricos se atribuyan a un autor corporativo. Todos los miembros del grupo que sean designados como autores, ya sea en la línea destinada al nombre de los autores a continuación del título o en una nota a pie de página, deberán cumplir plenamente con los requisitos de autoría recién señalados. Los miembros del grupo que no cumplan con dichos criterios serán mencionados, con su autorización, en la sección de agradecimientos o en un apéndice (véase "Agradecimientos").

El orden en que figuran los autores debe reflejar una decisión conjunta de estos. Como los autores se suelen enumerar de distintas maneras, el significado del orden en que aparecen no puede deducirse con exactitud a menos que ellos mismos lo enuncien explícitamente. Para tal efecto, tal vez deseen agregar, en una nota a pie de página, la explicación sobre el orden de enumeración. Al decidir acerca de dicho orden, los autores tendrán presente que muchas revistas imponen un límite al número de autores que figuran en el índice de materias y que, cuando hay más de 25 autores, la Biblioteca Nacional de Medicina de los Estados Unidos incluye en MEDLINE tan solo los nombres de los 24 primeros más el del último.

\section{Resumen y palabras clave}

La segunda página incluirá un resumen (que no sobrepasará las 150 palabras de extensión si es un resumen ordinario o las 250 si es uno estructurado). En él se indicarán los propósitos del estudio o investigación; los procedimientos básicos (selección de los sujetos o los animales de laboratorio incluidos en el estudio; métodos de observación y análisis); los hallazgos más importantes (proporciónense datos específicos y, de ser posible, su significación estadística); y las conclusiones principales. Hágase hincapié en los aspectos nuevos e importantes del estudio o las observaciones.

A continuación del resumen agréguense, debidamente rotuladas, de 3 a 10 palabras o frases cortas clave que ayuden a los indizadores a clasificar el artículo, las cuales se publicarán junto con el resumen. Utilícense para este propósito los términos de la lista "Medical Subject Headings" (MeSH) [Encabezamientos de temas médicos] del Index Medicus; en el caso de términos de reciente aparición que todavía no figuren en dicha lista, podrán usarse las expresiones corrientes.

\section{Introducción}

Exprese el propósito del artículo y resuma el fundamento lógico del estudio u observación. Mencione las referencias estrictamente pertinentes y no incluya datos ni conclusiones del trabajo que está dando a conocer.

\section{Métodos}

Describa claramente la forma como se seleccionaron los sujetos observados o que participaron en los experimentos (pacientes o animales de laboratorio, incluidos los testigos).

Identifique la edad, el sexo y otras características importantes de los sujetos. La definición y la pertinencia de la raza y el grupo étnico son ambiguos. Los autores deberán ser particularmente cuidadosos con respecto a usar estas categorías.

Identifique los métodos, los aparatos (nombre y dirección del fabricante entre paréntesis) y los procedimientos con detalles suficientes para que otros investigadores puedan reproducir los resultados. Proporcione referencias de los métodos acreditados, incluidos los de índole estadística (véase más adelante); dé referencias y explique brevemente los métodos ya publicados pero que no son bien conocidos; describa 
los métodos nuevos o que han sido sustancialmente modificados, manifestando las razones por las cuales se usaron y evaluando sus limitaciones. Identifique exactamente todos los medicamentos y productos químicos utilizados, sin olvidar nombres genéricos, dosis y vías de administración.

Los informes de ensayos clínicos aleatorizados deberán presentar información sobre todos los elementos importantes del estudio, como son el protocolo (población de estudio, intervenciones o exposiciones, resultados y el fundamento lógico del análisis estadístico), asignación de intervenciones (métodos de aleatorización, ocultamiento de la asignación a los grupos de tratamiento) y método de enmascaramiento (método ciego).

Los autores que presenten manuscritos de revisión incluirán una sección en la que se describan los métodos utilizados para localizar, seleccionar, extraer y sintetizar los datos. Estos métodos se mencionarán también en forma sinóptica en el resumen.

Ética. Cuando informe sobre experimentos en seres humanos, señale si los procedimientos seguidos estuvieron de acuerdo con las normas éticas del comité (institucional o regional) que supervisa la experimentación en seres humanos o con la Declaración de Helsinki de 1975, modificada en 1983. No utilice el nombre de los pacientes, sus iniciales ni los códigos hospitalarios, especialmente en el material ilustrativo. Cuando dé a conocer experimentos con animales, mencione si se cumplieron las normas de la institución, las de un consejo nacional de investigación o cualquier ley nacional acerca del cuidado y el uso de animales de laboratorio.

Estadística. Describa los métodos estadísticos con detalles suficientes para que el lector versado en el tema y que tenga acceso a los datos originales pueda verificar los resultados presentados. Siempre que sea posible, cuantifique los resultados y preséntelos con indicadores apropiados de error o incertidumbre de la medición (por ej., intervalos de confianza). No dependa exclusivamente de las pruebas de comprobación de hipótesis estadísticas, tales como el uso de los valores $P$, que no transmiten información cuantitativa importante. Analice la elegibilidad de los sujetos de experimentación. Proporcione los detalles del proceso de aleatorización. Describa los medios utilizados para enmascarar las observaciones (método ciego), indicando los resultados que dieron. Informe sobre las complicaciones del tratamiento. Especifique el número de observaciones. Mencione las pérdidas de sujetos de observación (por ej., las personas que abandonan un ensayo clínico). Siempre que sea posible, las referencias sobre el diseño del estudio y los métodos estadísticos utilizados serán de trabajos vigentes (indicando el número de las páginas), y no de los artículos originales donde se describieron por vez primera. Especifique cualquier programa de computación de uso general que se haya empleado.

Las descripciones generales de los métodos utilizados deben aparecer en la sección de métodos. Cuando resuma los datos en la sección de resultados, especifique los métodos estadísticos que se emplearon para analizarlos. Limite el número de cuadros y figuras al mínimo necesario para explicar el tema central del artículo y para evaluar los datos en que se apoya. Use gráficas en vez de cuadros subdivididos en muchas partes; no duplique los datos en las gráficas y los cuadros. Evite el uso no técnico de términos de la estadística, tales como "al azar" (que entraña el empleo de un método de aleatorización), "normal", "significativo", "correlaciones" y "muestra". Defina los términos, las abreviaturas y la mayor parte de los símbolos estadísticos.

\section{Resultados}

En el texto, los cuadros y las ilustraciones, presente los resultados siguiendo una secuencia lógica. No repita en el texto todos los datos de los cuadros ni de las ilustraciones; destaque o resuma tan solo las observaciones importantes.

\section{Discusión}

Haga hincapié en los aspectos nuevos e importantes del estudio y en las conclusiones que se derivan de ellos. No repita con pormenores los datos $u$ otra información ya presentados en las secciones de introducción y de resultados. Explique en la sección de discusión el significado de los hallazgos y sus limitaciones, incluidas sus implicaciones para la investigación futura. Relacione las observaciones con otros estudios pertinentes.

Establezca el nexo entre las conclusiones y los objetivos del estudio, pero absténgase de hacer afirmaciones generales y extraer conclusiones que no estén completamente respaldadas por los datos. En particular, los autores evitarán hacer afirmaciones sobre los beneficios y los costos económicos, a menos que su manuscrito incluya datos y análisis económicos. No reclame ningún tipo de precedencia ni mencione trabajos que no estén terminados. Proponga nuevas hipótesis cuando haya justificación para ello, pero identificándolas claramente como tales. Cuando sea apropiado, puede incluir recomendaciones.

\section{Agradecimientos}

En un lugar adecuado del artículo (como nota al pie de la primera página o como apéndice del texto; véanse los requisitos de la revista) uno o varios enunciados especificarán lo siguiente: 1) las colaboraciones que deben ser reconocidas pero que no justifican la autoría, tales como el apoyo general del jefe del departamento; 2) el reconocimiento por la ayuda técnica recibida; 3 ) el agradecimiento por el apoyo financiero y material, especificando la índole del mismo; y 4) las relaciones que puedan suscitar un conflicto de intereses (véase "Conflicto de intereses"). 
Las personas que colaboraron intelectualmente en el artículo pero cuya participación no justifica la autoría pueden ser citadas por su nombre, añadiendo su función o tipo de colaboración; por ejemplo, "asesoramiento científico", "examen crítico de la propuesta para el estudio", "recolección de los datos" o "participación en el ensayo clínico". Estas personas tendrán que conceder su permiso para ser nombradas. Los autores se responsabilizarán de obtener la autorización por escrito de las personas mencionadas por su nombre en los agradecimientos, pues los lectores pueden inferir que estas respaldan los datos y las conclusiones.

El reconocimiento por la ayuda técnica recibida figurará en un párrafo separado de los testimonios de gratitud por otras contribuciones.

\section{Referencias}

Numere las referencias consecutivamente siguiendo el orden en que se mencionan por primera vez en el texto. En este, en los cuadros y en los pies o epígrafes de las ilustraciones, las referencias se identificarán mediante números arábigos entre paréntesis. Las referencias citadas solamente en cuadros o ilustraciones se numerarán siguiendo una secuencia que se establecerá por la primera mención que se haga en el texto de ese cuadro o esa figura en particular.

Emplee el estilo de los ejemplos que aparecen más adelante, los cuales están basados en el formato que la Biblioteca Nacional de Medicina de los Estados Unidos usa en el Index Medicus. Abrevie los títulos de las revistas de conformidad con el estilo utilizado en dicha publicación. Consulte la List of Journals Indexed in Index Medicus [Lista de revistas indizadas en Index Medicus], que se publica anualmente como parte del número de enero $\mathrm{y}$ como separata. La lista se puede obtener asimismo en el sitio que la biblioteca mantiene en la World Wide Web (http:/ / www.nlm.nih.gov).

Absténgase de utilizar los resúmenes como referencias. Las referencias a artículos que han sido aceptados pero que todavía no se publican se designarán como "en prensa" o "de próxima aparición"; los autores obtendrán por escrito el permiso para citar dichos artículos y también la verificación de que han sido aceptados para publicación. La información proveniente de manuscritos presentados para publicación pero aún no aceptados se citará en el texto como "observaciones inéditas", con el permiso correspondiente de la fuente.

No cite una "comunicación personal" a menos que aporte información esencial que no pueda obtenerse de una fuente pública; en ese caso, el nombre de la persona y la fecha de la comunicación aparecerán entre paréntesis en el texto. En el caso de artículos científicos, los autores deberán obtener el permiso de la fuente y su confirmación de la exactitud de la comunicación personal, ambos por escrito.

Los autores verificarán las referencias cotejándolas contra los documentos originales.

El estilo de los requisitos uniformes (estilo de Vancouver) se basa en gran medida en una norma de estilo ANSI adaptada por la Biblioteca Nacional de Medicina (NLM) para sus bases de datos. En los ejemplos que siguen se han agregado notas cuando el estilo de Vancouver difiere del estilo que actualmente utiliza la NLM.

\section{Artículos de revista}

\section{Artículo de revista ordinario}

Enumere los primeros seis autores y añada la expresión "et al."

(Nota: La NLM incluye ahora hasta 25 autores; si hay más de 25 , enumera los primeros 24, a continuación el último autor y luego agrega "et al.")

Vega KJ, Pina I, Krevsky B. Heart transplantation is associated with an increased risk for pancreatobiliary disease. Ann Intern Med 1996 Jun 1;124(11):980-3.

Optativamente, si se utiliza la paginación continua a lo largo de un volumen (como hacen muchas revistas médicas), se pueden omitir el mes y el número.

(Nota: Para respetar la uniformidad, en todos los ejemplos que se presentan en los requisitos uniformes se aplica esta opción. La NLM, sin embargo, no usa dicha opción.)

Vega KJ, Pina I, Krevsky B. Heart transplantation is associated with an increased risk for pancreatobiliary disease. Ann Intern Med 1996;124:980-3.

Más de seis autores:

Parkin DM, Clayton D, Black RJ, Masuyer E, Friedl HP, Ivanov E, et al. Childhood leukaemia in Europe after Chernobyl: 5 year follow-up. Br J Cancer 1996;73:1006-12.

\section{Organización como autor}

The Cardiac Society of Australia and New Zealand. Clinical exercise stress testing. Safety and performance guidelines. Med J Aust 1996;164:282-4.

\section{No se indica el nombre del autor}

Cancer in South Africa [editorial]. S Afr Med J 1994;84:15.

\section{Artículo en idioma extranjero ${ }^{3}$}

(Nota: La NLM traduce el título al inglés, lo encierra entre corchetes y le agrega la abreviatura correspondiente al idioma original.)

Ryder TE, Haukeland EA, Solhaug JH. Bilateral infrapatellar seneruptur hos tidligere frisk kvinne. Tidsskr Nor Laegeforen 1996; 116:41-2.

\section{Suplemento de un volumen}

Shen HM, Zhang QF. Risk assessment of nickel carcinogenicity and occupational lung cancer. Environ Health Perspect 1994;102 Suppl 1:275-82.

\section{Suplemento de un número}

Payne DK, Sullivan MD, Massie MJ. Women's psychological reactions to breast cancer. Semin Oncol 1996;23(1 Suppl 2): 89-97.

\section{Parte de un volumen}

Ozben T, Nacitarhan S, Tuncer N. Plasma and urine sialic acid in non-insulin dependent diabetes mellitus. Ann Clin Biochem 1995;32(Pt 3):303-6.

\section{Parte de un número}

Poole GH, Mills SM. One hundred consecutive cases of flap lacerations of the leg in ageing patients. N Z Med J 1994;107(986 Pt 1):377-8.

\section{Número sin volumen}

Turan I, Wredmark T, Fellander-Tsai L. Arthroscopic ankle arthrodesis in rheumatoid arthritis. Clin Orthop 1995;(320): 110-4.

\footnotetext{
3 Evidentemente, "extranjero" se entiende aquí en relación con el idioma inglés, pues los ejemplos de referencias bibliográficas se han trasladado directamente del original, sin adaptarlos. [N. del t.]
} 


\section{Sin número ni volumen}

Browell DA, Lennard TW. Immunologic status of the cancer patient and the effects of blood transfusion on antitumor responses. Curr Opin Gen Surg 1993:325-33.

\section{Paginación en números romanos}

Fisher GA, Sikic BI. Drug resistance in clinical oncology and hematology. Introduction. Hematol Oncol Clin North Am 1995 Apr;9(2):xi-xii.

12. Indicación del tipo de artículo, según corresponda

Enzensberger W, Fischer PA. Metronome in Parkinson's disease [carta]. Lancet 1996;347: 1337.

Clement J, De Bock R. Hematological complications of hantavirus nephropathy (HVN) [resumen]. Kidney Int 1992;42:1285.

\section{Artículo que contiene una retracta-} ción

Garey CE, Schwarzman AL, Rise ML, Seyfried TN. Ceruloplasmin gene defect associated with epilepsy in EL mice [retractación de Garey CE, Schwarzman AL, Rise ML, Seyfried TN. En: Nat Genet 1994;6:426-31]. Nat Genet 1995;11:104.

\section{Artículo retirado por retractación}

Liou GI, Wang M, Matragoon S. Precocious IRBP gene expression during mouse development [retirado por retractación en Invest Ophthalmol Vis Sci 1994;35:3127]. Invest Ophthalmol Vis Sci 1994;35:1083-8.

15. Artículo sobre el que se ha publicado una fe de erratas

Hamlin JA, Kahn AM. Herniography in symptomatic patients following inguinal hernia repair [se publica una fe de erratas en West J Med 1995;162:278]. West J Med 1995; 162:28-31.

\section{Libros y otras monografías}

(Nota: Con anterioridad, el estilo de Vancouver indicaba, incorrectamente, que entre la editorial y la fecha debía ir una coma en vez de punto y coma, como debe ser.)

\section{Individuos como autores}

Ringsven MK, Bond D. Gerontology and leadership skills for nurses. 2nd ed. Albany (NY): Delmar Publishers; 1996

\section{Directores ("editores"), compiladores como autores}

Norman IJ, Redfern SJ, editors. Mental health care for elderly people. New York: Churchill Livingstone; 1996.

\section{Organización como autor y editorial}

Institute of Medicine (US). Looking at the future of the Medicaid program. Washington: The Institute; 1992.

19. Capítulo de libro

(Nota: Con anterioridad, el estilo de Vancouver prescribía el uso de dos puntos en vez de la letra $p$ antes de las páginas.)

Phillips SJ, Whisnant JP. Hypertension and stroke. En: Laragh JH, Brenner BM, editors. Hypertension: pathophysiology, diagnosis, and management. 2nd ed. New York: Raven Press; 1995. p. 465-78.

\section{Actas de conferencias}

Kimura J, Shibasaki H, editors. Recent advances in clinical neurophysiology. Proceedings of the 10th International Congress of EMG and Clinical Neurophysiology; 1995 Oct 15-19; Kyoto, Japan. Amsterdam: Elsevier; 1996.

\section{Artículo presentado en una conferen-} cia

Bengtsson S, Solheim BG. Enforcement of data protection, privacy and security in medical informatics. En: Lun KC, Degoulet $\mathrm{P}$, Piemme TE, Rienhoff $\mathrm{O}$, editors. MEDINFO 92. Proceedings of the 7th World Congress on Medical Informatics; 1992 Sep 6-10; Geneva, Switzerland. Amsterdam: North-Holland; 1992. p. 1561-5.

\section{Informe científico o técnico}

Publicado por la institución financiadora o patrocinadora:

Smith P, Golladay K. Payment for durable medical equipment billed during skilled nursing facility stays. Final report. Dallas (TX): Dept. of Health and Human Services (US),

Office of Evaluation and Inspections; 1994 Oct. Report No.: HHSIGOEI69200860.

Publicado por la institución ejecutora:

Field MJ, Tranquada RE, Feasley JC, editors. Health services research: work force and educational issues. Washington: National Academy Press; 1995. Contract No.: AHCPR282942008. Sponsored by the Agency for Health Care Policy and Research.

\section{Tesis doctoral}

Kaplan SJ. Post-hospital home health care: the elderly's access and utilization [tesis doctoral]. St. Louis (MO): Washington Univ.; 1995.

\section{Patente}

Larsen CE, Trip R, Johnson CR, inventors; Novoste Corporation, titular. Methods for procedures related to the electrophysiology of the heart. US patent 5,529,067. 1995 Jun 25.

\section{Otros trabajos publicados}

\section{Artículo de periódico}

Lee G. Hospitalizations tied to ozone pollution: study estimates 50,000 admissions annually. The Washington Post 1996 Jun 21; Sect. A:3 (col. 5).

\section{Material audiovisual}

HIV+/AIDS: the facts and the future [videocassette]. St. Louis (MO): Mosby-Year Book; 1995.

27. Documentos legales

Ley pública:

Preventive Health Amendments of 1993 Pub. L. No. 103-183, 107 Stat. 2226 (Dec. 14, 1993).

Proyecto de ley sin sancionar:

Medical Records Confidentiality Act of 1995 S. 1360, 104th Cong., 1st Sess. (1995).

Código de normas federales: Informed Consent, 42 C.F.R. Sect. 441.257 (1995).

Audiencia

Increased Drug Abuse: the Impact on the Nation's Emergency Rooms: Hearings Before the Subcomm. on Human Resources and Intergovernmental Relations of the House Comm. on Government Operations, 103rd Cong., 1st Sess. (May 26, 1993).

\section{Mapa}

North Carolina. Tuberculosis rates per 100,000 population, 1990 [mapa demográfico]. Raleigh: North Carolina Dept. of Environment, Health, and Natural Resources, Div. of Epidemiology; 1991.

\section{Libro de la Biblia}

The Holy Bible. King James version. Grand Rapids (MI): Zondervan Publishing House; 1995. Ruth 3:1-18.

30. Diccionarios y obras de consulta semejantes

Stedman's medical dictionary. 26th ed. Baltimore: Williams \& Wilkins; 1995. Apraxia; p. 119-20.

\section{Obras clásicas}

The Winter's Tale: act 5, scene 1, lines 13-16. The complete works of William Shakespeare. London: Rex; 1973.

\section{Trabajos inéditos}

\section{En prensa}

(Nota: La NLM prefiere referirse a estos trabajos como "en preparación" [forthcoming] porque no todos se publicarán impresos.) 
Leshner AI. Molecular mechanisms of cocaine addiction. N Engl J Med. En prensa 1996.

\section{Material en soporte electrónico}

\section{Artículo de revista en formato electró-} nico

Morse SS. Factors in the emergence of infectious diseases. Emerg Infect Dis [publicación periódica en línea] 1995 Jan-Mar [citada 1996 Jun 5];1(1):[24 pantallas]. Se consigue en: URL: http://www.cdc.gov/ncidod/EID/ eid.htm

\section{Monografía en formato electrónico}

CDI, clinical dermatology illustrated [monografía en CD-ROM]. Reeves JRT, Maibach $\mathrm{H}$. CMEA Multimedia Group, producers. 2nd ed. Version 2.0. San Diego: CMEA; 1995.

\section{Fichero de computadora}

Hemodynamics III: the ups and downs of hemodynamics [programa de computadora]. Version 2.2. Orlando (FL): Computerized Educational Systems; 1993.

\section{Cuadros}

Mecanografíe o imprima cada cuadro a doble espacio y en hoja aparte. No presente los cuadros en forma de impresiones fotográficas. Numérelos consecutivamente siguiendo el orden en que se citan por primera vez en el texto, y asigne un título breve a cada uno. Cada columna llevará un encabezamiento corto o abreviado. Las explicaciones irán como notas al pie y no en el encabezamiento. En las notas al pie se explicarán todas las abreviaturas no usuales empleadas en cada cuadro. Como llamadas para las notas al pie, utilícense los símbolos siguientes en la secuencia que se indica: ${ }^{*}, \dagger, \ddagger, \S, \|, \mathbb{I}$,

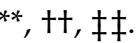

Identifique las medidas estadísticas de variación, tales como la desviación estándar y el error estándar de la media.

No trace líneas horizontales ni verticales en el interior de los cuadros.

Cerciórese de que cada cuadro aparezca citado en el texto.

Si incluye datos publicados o inéditos provenientes de otra fuente, obtenga la autorización necesaria para reproducirlos y conceda el reconocimiento cabal que corresponde.
Incluir un número excesivo de cuadros en relación con la extensión del texto puede ocasionar dificultades al confeccionar las páginas. Examine varios números recientes de la revista a la que planea presentar el artículo y calcule cuántos cuadros pueden incluirse por cada millar de palabras de texto.

Al aceptar un artículo, el director podrá recomendar que los cuadros suplementarios que contienen datos de respaldo importantes, pero que son muy extensos para publicarlos, queden depositados en un servicio de archivo, como el Servicio Nacional de Publicaciones Auxiliares en los Estados Unidos, o que sean proporcionados por los autores a quien lo solicite. En tal caso, se agregará en el texto la nota informativa necesaria. Dichos cuadros se presentarán junto con el artículo para su consideración.

\section{Ilustraciones (figuras)}

Envíe los juegos completos de figuras en el número requerido por la revista. Las figuras estarán dibujadas y fotografiadas en forma profesional; no se aceptarán los letreros trazados a mano o con máquina de escribir. En lugar de los dibujos, radiografías y otros materiales de ilustración originales, envíe impresiones fotográficas en blanco y negro, bien contrastadas, en papel satinado y que midan $127 \times 173$ $\mathrm{mm}$, sin exceder de $203 \times 254 \mathrm{~mm}$. Las letras, números y símbolos serán claros y uniformes en todas las ilustraciones; tendrán, además, un tamaño suficiente para que sigan siendo legibles incluso después de la reducción necesaria para publicarlos. Los títulos y las explicaciones detalladas se incluirán en los pies o epígrafes, no sobre las propias ilustraciones.

Al reverso de cada figura pegue una etiqueta de papel que lleve anotados el número de la figura, el nombre del autor y cuál es la parte superior de la misma. No escriba directamente sobre el dorso de las figuras ni las sujete con broches para papel, pues quedan marcadas. Las figuras no se doblarán ni se montarán sobre cartón.
Las fotomicrografías incluirán en sí mismas un indicador de la escala. Los símbolos, flechas y letras usados en estas deberán contrastar claramente con el fondo.

Si se usan fotografías de personas, estas no deberán ser identificables; de lo contrario, habrá que anexar un permiso por escrito para poder utilizarlas (véase la sección "Protección del derecho de los pacientes a que se respete su vida privada").

Las figuras se numerarán en forma consecutiva de acuerdo con su primera mención en el texto. Si la figura ya fue publicada, se reconocerá la fuente original y se presentará la autorización por escrito que el titular de los derechos de autor concede para reproducirla. Este permiso es necesario, independientemente de quién sea el autor o la editorial; la única salvedad son los documentos considerados como de dominio público.

En el caso de las ilustraciones en color, averigüe si la revista necesita negativos, transparencias en positivo o impresiones fotográficas. La inclusión de un diagrama en el que se indique la parte de la fotografía que debe reproducirse puede resultar útil a la redacción. Algunas revistas publican ilustraciones en color únicamente si el autor paga el costo extra.

\section{Pies o epígrafes de las ilustraciones}

Los pies o epígrafes de las ilustraciones se mecanografiarán o imprimirán a doble espacio, comenzando en hoja aparte e identificándolos con los números arábigos correspondientes. Cuando se utilicen símbolos, flechas, números o letras para referirse a ciertas partes de las ilustraciones, será preciso identificar y aclarar el significado de cada uno en el pie o epígrafe. En las fotomicrografías habrá que explicar la escala y especificar el método de tinción.

\section{Unidades de medida}

Las medidas de longitud, talla, peso y volumen se expresarán en unidades del sistema métrico decimal (metro, 
kilogramo, litro, etc.) o sus múltiplos y submúltiplos.

Las temperaturas se consignarán en grados Celsius. Los valores de presión arterial se indicarán en milímetros de mercurio.

Todos los valores hemáticos y de química clínica se presentarán en unidades del sistema métrico decimal y de acuerdo con el Sistema Internacional de Unidades (SI). La redacción de la revista podrá solicitar que, antes de publicar el artículo, los autores agreguen unidades alternativas o distintas de las del SI.

\section{Abreviaturas y símbolos}

Utilice únicamente abreviaturas corrientes. Evite las abreviaturas en el título y el resumen. Cuando se emplee por primera vez una abreviatura en el texto, irá precedida del término completo, salvo si se trata de una unidad de medida común.

\section{ENVÍO DEL MANUSCRITO A LA REVISTA}

Envíe por correo el número requerido de copias del manuscrito en un sobre de papel resistente; si es necesario, proteja las copias y las figuras metiéndolas entre dos hojas de cartón para evitar que las fotografías se doblen. Meta las fotografías y transparencias en su propio sobre de papel resistente.

Los manuscritos irán acompañados de una carta de envío firmada por todos los coautores. Es preciso incluir en ella lo siguiente: 1) información acerca de la publicación previa o duplicada, o sobre la presentación de cualquier parte del trabajo a otra revista, según lo expresado líneas arriba; 2) una manifestación de las relaciones financieras o de otro tipo que pudieran dar lugar a un conflicto de intereses (véase más adelante); 3) una declaración de que el manuscrito ha sido leído y aprobado por todos los autores, que se ha cumplido con los requisitos de la autoría expuestos anteriormente en el presente documento y que cada autor está convencido de que el manuscrito representa un trabajo honrado; y 4) el nombre, la dirección y el número telefónico del autor corresponsal, quien se encargará de comunicarse con los demás autores en lo concerniente a las revisiones y a la aprobación final de las pruebas de imprenta. La carta incluirá cualquier información suplementaria que pueda resultar útil para el director, tal como el tipo de artículo que el manuscrito representa para esa revista en particular y si el autor (o los autores) estaría dispuesto a sufragar el costo de reproducir las ilustraciones en color.

El manuscrito se acompañará de copias de los permisos concedidos para reproducir material ya publicado, para usar ilustraciones o revelar información sobre individuos que puedan ser identificados, o para agradecer a ciertas personas su colaboración.

ABSTRACT A small group of editors of general medical journals met informally in Vancouver, British Columbia, in 1978 to establish guidelines for the format of manuscripts submitted to their journals. The group became known as the Vancouver Group. Its requirements for manuscripts,

Uniform Requirements for Manuscripts Submitted to Biomedical Journals including formats for bibliographic references developed by the National Library of Medicine, were first published in 1979. The Vancouver Group expanded and evolved into the International Committee of Medical Journal Editors (ICMJE), which meets annually; gradually it has broadened its concerns.

The committee has produced five editions of the Uniform Requirements for Manuscripts Submitted to Biomedical Journals. Over the years, issues have arisen that go beyond manuscript preparation. Some of these issues are now covered in the Uniform Requirements; others are addressed in separate statements. Each statement has been published in a scientific journal. 